\title{
Intelligent Prediction of Sieving Efficiency in Vibrating Screens
}

\author{
Bin Zhang, ${ }^{1,2}$ Jinke Gong, ${ }^{1}$ Wenhua Yuan, ${ }^{2}$ Jun Fu, ${ }^{2}$ and Yi Huang' \\ ${ }^{1}$ The State Key Laboratory of Advanced Design and Manufacturing for Vehicle Body, Hunan University, Changsha 410082, China \\ ${ }^{2}$ Department of Mechanical and Energy Engineering, Shaoyang University, Shaoyang 422004, China \\ Correspondence should be addressed to Jinke Gong; gongjinke@126.com
}

Received 25 November 2015; Revised 22 February 2016; Accepted 15 March 2016

Academic Editor: Longjun Dong

Copyright (C) 2016 Bin Zhang et al. This is an open access article distributed under the Creative Commons Attribution License, which permits unrestricted use, distribution, and reproduction in any medium, provided the original work is properly cited.

\begin{abstract}
In order to effectively predict the sieving efficiency of a vibrating screen, experiments to investigate the sieving efficiency were carried out. Relation between sieving efficiency and other working parameters in a vibrating screen such as mesh aperture size, screen length, inclination angle, vibration amplitude, and vibration frequency was analyzed. Based on the experiments, least square support vector machine (LS-SVM) was established to predict the sieving efficiency, and adaptive genetic algorithm and cross-validation algorithm were used to optimize the parameters in LS-SVM. By the examination of testing points, the prediction performance of least square support vector machine is better than that of the existing formula and neural network, and its average relative error is only $4.2 \%$.
\end{abstract}

\section{Introduction}

Sieving is one of the oldest physical size separation methods and has been widely used both in industries and in laboratories. Vibrating screens which include a number of types are the main sieving tool for large-scale separation and classification of solid particles by size, and they are widely used in some practical engineering such as mining, metallurgy, dry mortar, artificial sand, and agriculture production. Probability screen is a special vibrating screen for the separation and classification of fine particulate material, which exhibits problems of plugging holes and low sieving efficiency when the particle size is below $0.6 \mathrm{~mm}$. Sieving efficiency is an essential evaluation indicator of sieving performance, and it is hard to be predicted based on the existing sieving design parameters in the design process of vibrating screens due to the comprehensive effect of complex particle sieving process under multiple factors, which will influence the selection or determination of these parameters. Therefore, an understanding of predicting sieving efficiency has a great practical significance.

At present, the research on the sieving efficiency of vibrating screens has also made some progress. There are lots of researchers studying the sieving process by DEM simulations like Li et al. [1], Dong et al. [2], Liu [3], Delaney et al. [4], Jiao and Zhao [5], and Li et al. [6], and qualitative relation between sieving efficiency and sieving parameters in a vibrating screen such as amplitude, vibration frequency, screen mesh size, particle size, and vibration direction angle has been analyzed, which provides references for in-depth study. But the results of DEM simulations need to be further explored and improved since particulate materials and boundary conditions of simulation are difficult to coincide with the actual conditions. Some scholars have studied the real-time monitoring of sieving efficiency in the working course of a vibrating screen by gathering its vibration signals, but their research achievements only play the role of realtime monitoring and have little effect on the design of a vibrating screen [7]. In terms of sieving efficiency fitting, Grozubinsky et al. [8] and Chen and Tong [9] have, respectively, established formulas between the sieving efficiency and sieving parameters such as amplitude, vibration frequency, vibration direction angle, particle size, and screen mesh size based on a probabilistic model and a discrete element model, but these formulas only reflect the relationship between the single parameter and sieving efficiency. Jiao et al. [10] found the mathematical formula between sieving efficiency and parameters including screen deck angle and screen mesh size based on statistical analysis of experimental data, which provides a theoretical basis for the design of vibrating screens 
but ignores the impact of screen length. Although some fitting function formulas of sieving efficiency have been studied, there is still no widely accepted formula to predict sieving efficiency on the basis of sieving parameters. The introduction of artificial intelligence may provide a good path to the solution of this problem [11].

Support vector machine (SVM) and neural network both can fit the nonlinear relations [12-14], whereas SVM is more suitable when the sample size is relatively small and can solve "curse of dimensionality" problems. The solution of "curse of dimensionality" can make the complexity of algorithm and the dimension of sample independent. At present, SVM has been widely and effectively used in the pattern recognition, intelligent fitting, and prediction [1518]. However, the application of SVM to predict the sieving efficiency has not been reported in the literatures yet.

In this paper, the experimental system and results were firstly introduced and analyzed, and then intelligent fitting model of least square support vector machine (LS-SVM) and adaptive genetic algorithm were provided; finally, the contrast between the performance of the LS-SVM model, the existing formula, and the neural network was carried out.

\section{Sieving Experimental System}

Sieving is a process in which a certain size range of materials is separated into several products with different size through one-deck or multideck screens that have sieving mesh with uniform apertures. Theoretically, the particles whose size is larger than the mesh aperture remain on the screen surface and leave the screen surface when they pass the end of the screen, and these particles are called overflow particles; however, other smaller particles penetrate the sieving mesh through the mesh aperture and are called undersize particles. Sieving efficiency is the ratio between actual mass of undersize particles and the mass of the particles in raw materials whose size is smaller than the mesh aperture. Compared with the mesh aperture size, the smaller the particles are, the easier the penetration is, but the particles whose size is close to the mesh aperture size penetrate the screen mesh with difficulty. Probability screens have some advantages in all vibrating screens such as large sieving capacity and easy penetration because of their unique characteristics of the large mesh aperture and large inclination angle.

The repeated sieving tests are finished in an experimental system, which is illustrated in Figure 1. Sieving parameters such as inclination angle, screen length, mesh aperture size, amplitude, and vibration frequency are adjustable in this vibration sieving test bench, which is a circular experimental system and includes the multideck probability screen, storage bin of raw materials, vibrating feeder, and automatic feeding system. Samples used for measuring the sieving efficiency by Hancock's total efficiency formula are gotten when the screen is in steady working condition. The "steady working condition" means that the screen works steadily after starting the screen on the sieving test bench; meanwhile, raw materials cover the whole screen mesh surface and the feed rate of raw materials from vibrating feeder reaches

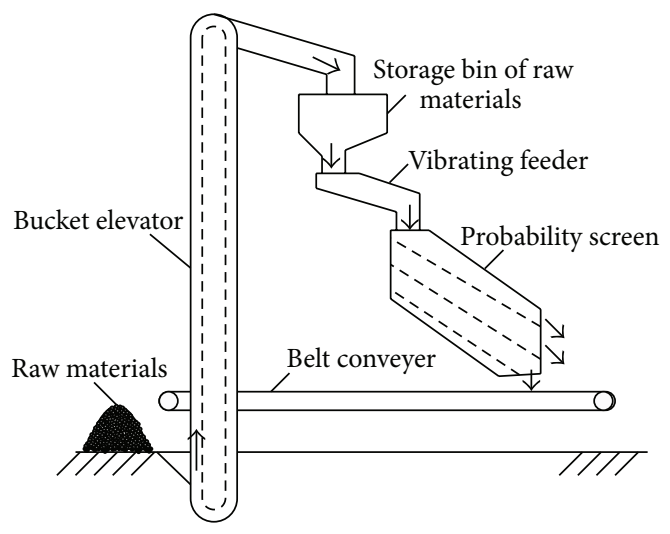

Figure 1: Schematic diagram of the vibration sieving test bench.

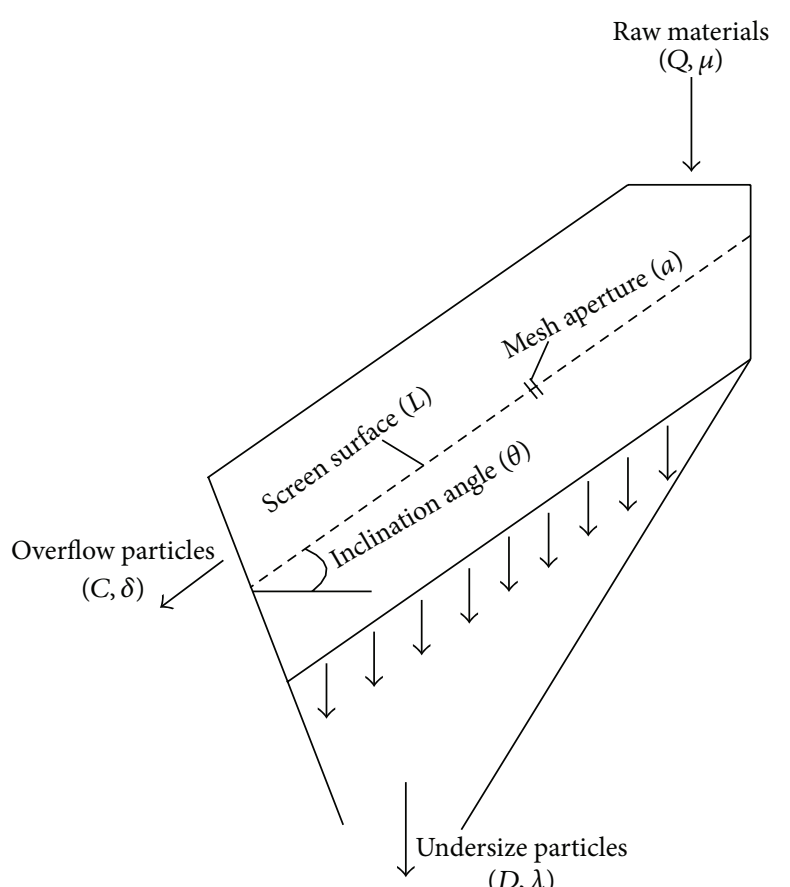

FIgURE 2: Schematic diagram of total efficiency calculation.

a stable state. Total efficiency is the difference between the penetration probability of the particles whose size is smaller than separation size and the penetration probability of the particles whose size is larger than separation size. The schematic diagram of total efficiency calculation is shown in Figure 2.

In Figure 2, $Q$ is the total mass of raw materials into the screen, $C$ is the mass of overflow particles, $D$ is the mass of undersize particles, $\mu$ is the percentage of the particles whose size is smaller than separation size in raw materials, $\delta$ is the percentage of the particles whose size is smaller than separation size in overflow particles, $\lambda$ is the percentage of the particles whose size is smaller than separation size in undersize particles, $a$ is mesh aperture size of the screen mesh, 
TABLE 1: Sand properties and operating conditions.

\begin{tabular}{lc}
\hline Parameters & Actual values \\
\hline Sand size $/ \mathrm{mm}$ & $0-4.75$ \\
Moisture ratio & $0.3 \%$ \\
Fineness modulus & 2.68 \\
Sieving capacity/(t/h) & 50 \\
\hline
\end{tabular}

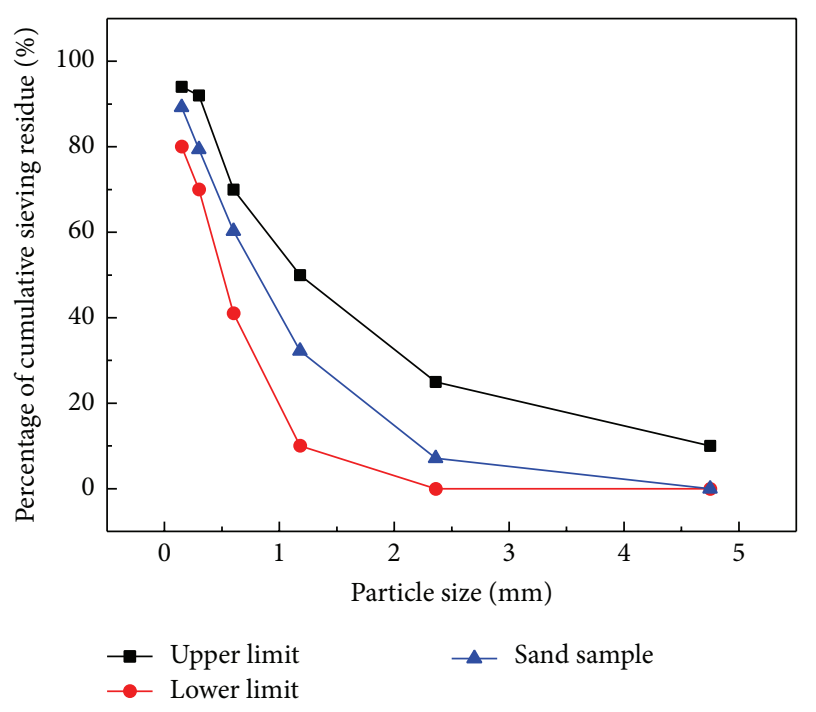

FIGURE 3: Grading curves of sands.

$L$ is screen length, and $\theta$ is inclination angle of the screen surface. Total efficiency is calculated as follows [10]:

$$
\eta_{\text {total }}=\frac{(\mu-\delta)(\lambda-\mu)}{\mu(\lambda-\delta)(100-\mu)} \times 100 \% .
$$

In test process, the screen mesh with rectangular apertures which is woven by steel wire is selected, and the common II area sands in construction industry whose particle diameter is between thick sands and fine sands are selected as raw materials. Sand properties and operating conditions are shown in Table 1, and grading curves of sands are shown in Figure 3.

As is shown in Figure 3, the particle diameter corresponding to the 6 data points is $0.15 \mathrm{~mm}, 0.3 \mathrm{~mm}, 0.6 \mathrm{~mm}, 1.18 \mathrm{~mm}$, $2.36 \mathrm{~mm}$, and $4.75 \mathrm{~mm}$; the grading curve of the sand sample is almost in the middle position between the upper and lower limits of the common sand. Since the raw sands are shifted with this multideck probability screen whose separation size is $2.36 \mathrm{~mm}, 1.18 \mathrm{~mm}$, and $0.6 \mathrm{~mm}$, respectively, from top to bottom, and, generally, the $0.6 \mathrm{~mm}$ layer of this probability screen has a smallest sieving efficiency, which sieves finest materials with the particle diameter below $0.6 \mathrm{~mm}$, the sieving efficiency of the $0.6 \mathrm{~mm}$ layer is viewed as the probability screen's one.

\section{Experimental Results}

According to experimental results, the sieving efficiency $\eta$ first increases with the increase of vibration

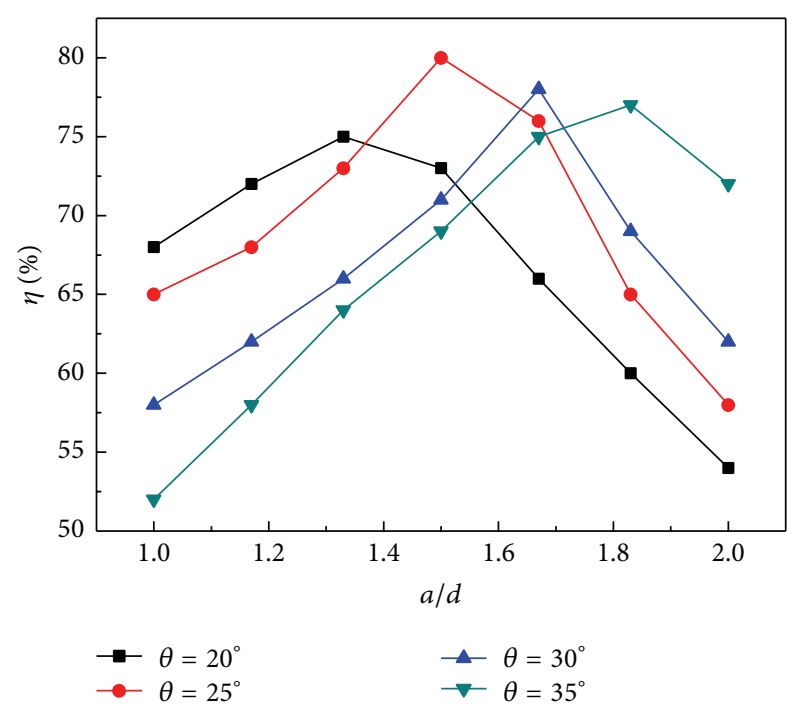

FIgURE 4: Effect of mesh aperture size on the sieving efficiency.

amplitude $A_{m}$ and screen length $L$ and then decreases with the increase of vibration frequency $\omega$, mesh aperture size $a$, and inclination angle $\theta$. This paper focuses on detailed analysis of $a, L$, and $\theta$, and the effect of $A_{m}$ and $\omega$ on the sieving efficiency is detailed in literature [7].

3.1. Effect of Mesh Aperture Size on the Sieving Efficiency. The mesh aperture size can also be characterized by the ratio between mesh aperture size $a$ and separation size $d$. $a / d$ is greater than 1 in probability screens. Figure 4 shows the effect of mesh aperture size $a$ on the sieving efficiency when $A_{m}$ is equal to $3.5 \mathrm{~mm}, \omega$ is equal to $960 \mathrm{r} / \mathrm{min}$, and $L$ is equal to $2.4 \mathrm{~m}$. $\eta$ first increases and then decreases with the increase of $a$. When the mesh aperture size is small, the sieving efficiency is low due to the approximate size between $a$ and $d$ and the lower penetration probability of the fine particles and a greater proportion of fine particles remained in overflow particles. When the mesh aperture size is larger, the sieving efficiency is higher due to the larger penetration probability of the fine particles. When the mesh aperture size is too large, the sieving efficiency becomes lower due to the higher penetration probability of the particles whose size is larger than separation size and undersize particles mixed with a greater proportion of larger particles. The optimum mesh aperture size is between $0.8 \mathrm{~mm}$ and $1.1 \mathrm{~mm}$, and the maximum efficiency can reach above $80 \%$ when $\theta$ is $25^{\circ}$.

3.2. Effect of Screen Length on the Sieving Efficiency. Figure 5 shows the effect of screen length $L$ on the sieving efficiency when $A_{m}$ is equal to $3 \mathrm{~mm}, \omega$ is equal to $960 \mathrm{r} / \mathrm{min}$, and $\theta$ is equal to $25^{\circ} . \eta$ increases significantly with the increase of $L$, and the increase of $\eta$ becomes slow after $L$ reaches a certain value. However, $\eta$ decreases with the further increase of $L$ under the condition of larger value of $a / d$ due to the high penetration probability of the particles whose size is larger than separation size and undersize particles mixed with an increasing number of impurities. If $a / d$ is larger, the optimum 


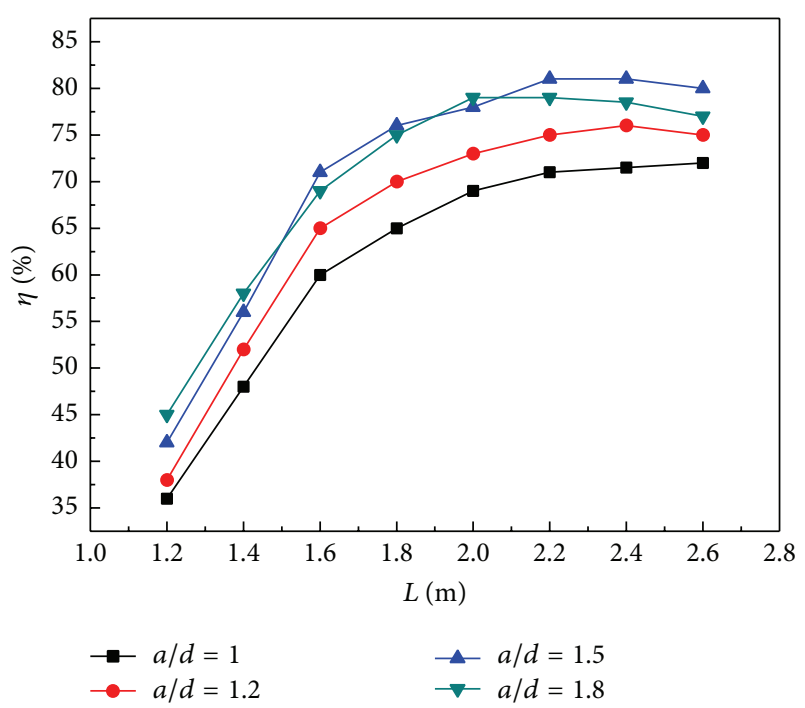

FiguRE 5: Effect of screen length on the sieving efficiency.

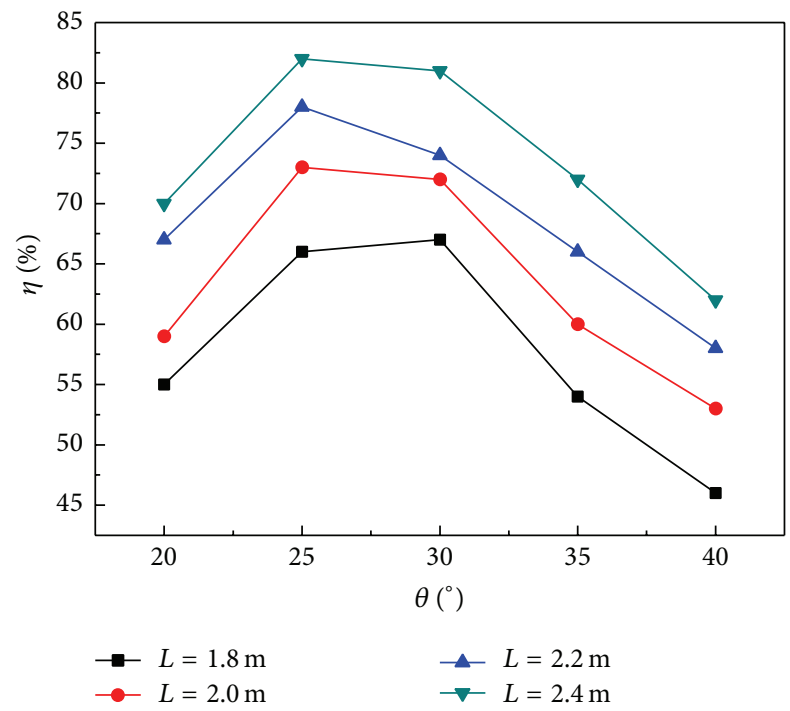

FIGURE 6: Effect of inclination angle on the sieving efficiency.

$L$ is shorter due to the high penetration probability of the particles whose size is smaller than separation size during the beginning time of sieving. The optimum range of screen length is between $2.0 \mathrm{~m}$ and $2.6 \mathrm{~m}$.

3.3. Effect of Inclination Angle on the Sieving Efficiency. Inclination angle $\theta$ mainly affects the horizontal projection size of mesh aperture and the moving speed of particles on the screen surface. Figure 6 shows the effect of inclination angle $\theta$ on the sieving efficiency when $A_{m}$ is equal to $3.5 \mathrm{~mm}, \omega$ is equal to $960 \mathrm{r} / \mathrm{min}$, and $a$ is equal to $0.9 \mathrm{~mm}$. $\eta$ first increases and then decreases with the increase of $\theta$, and $\eta$ reaches the maximum value with a certain inclination angle. When the inclination angle increases, the horizontal projection size of mesh aperture decreases and penetration probability of

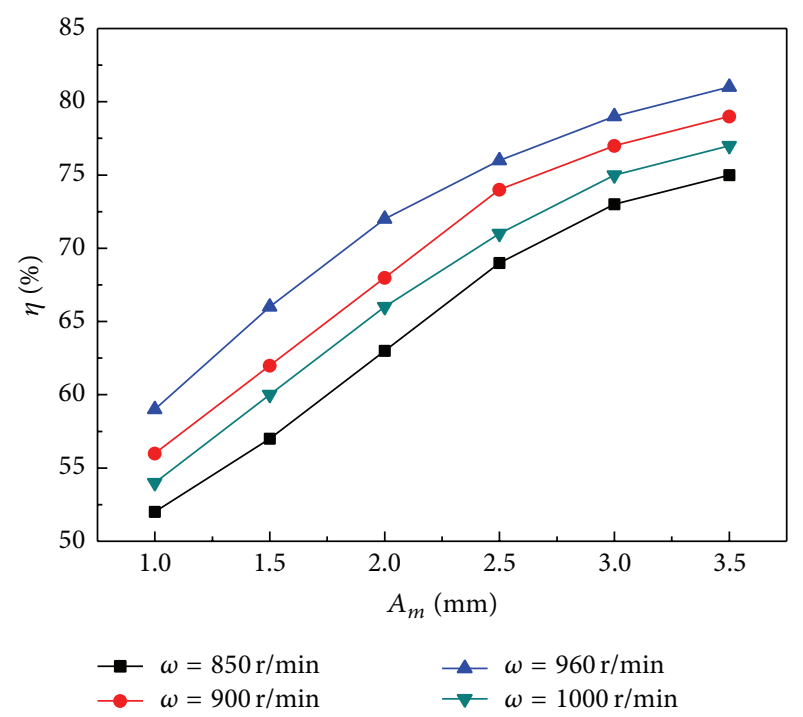

Figure 7: Effect of vibration amplitude on the sieving efficiency.

particles in the vertical direction decreases, which results in a lower penetration amount of the particles whose size is larger than separation size. Meanwhile, penetration and stratification capacity of the particles whose size is smaller than separation size is enhanced with increasing moving speed of particles on the screen surface. When the inclination angle increases further, the sieving efficiency becomes low due to sharp decrease of the horizontal projection size of mesh aperture, sharp increase of the moving speed of particles on the screen surface, and shorter stay time of particles on the screen surface. The optimum inclination angle is about $25^{\circ}$ and the maximum efficiency can reach above $82 \%$ when $L$ is equal to $2.4 \mathrm{~m}$.

3.4. Effect of Vibration Amplitude on the Sieving Efficiency. Figure 7 shows the effect of vibration amplitude $A_{m}$ on the sieving efficiency when $a$ is equal to $0.9 \mathrm{~mm}, L$ is equal to $2.4 \mathrm{~m}$, and $\theta$ is equal to $25^{\circ} . \eta$ increases with the increase of $A_{m}$, and $\eta$ first increases and then decreases with the increase of $\omega$. When $A_{m}$ and $\omega$ increase, the penetration probability of particles whose size is smaller than separation size becomes larger, so the sieving efficiency increases. However, when $\omega$ increases to $1000 \mathrm{r} / \mathrm{min}, \eta$ decreases because larger vibration frequency can lead to larger penetration probability of particles whose size is larger than separation size. Optimum vibration amplitude and vibration frequency are $3.5 \mathrm{~mm}$ and $960 \mathrm{r} / \mathrm{min}$, respectively.

\section{Establishment of the Intelligent Model Based on LS-SVM}

It is supposed that there are lots of training points $H=$ $\left\{x_{i}, y_{i}\right\}(i=1,2, \ldots, n)\left(x_{i}\right.$ is the input value, $y_{i}$ is the output value, and $n$ is the total number of training points) to be fitted; 
according to the basic theory of SVM, these problems can be converted to the solution of the following equations:

$$
\begin{array}{ll}
\max \quad\left\{a_{j}\right\}\left\{a_{j}^{*}\right\} & \\
= & -\frac{1}{2} \sum_{i=1}^{n} \sum_{j=1}^{n}\left(a_{i}-a_{i}^{*}\right)\left(a_{j}-a_{j}^{*}\right) k\left(x_{i}, x_{j}\right) \\
& -\varepsilon \sum_{i=1}^{n}\left(a_{i}+a_{i}^{*}\right)+\sum_{i=1}^{n} y_{i}\left(a_{i}-a_{i}^{*}\right) \\
\text { s.t. } \quad \sum_{i=1}^{n}\left(a_{i}-a_{i}^{*}\right)=0 & a_{i}, a_{i}^{*} \in[0, c] \\
f(x)= & \sum_{i=1}^{n}\left(a_{i}-a_{i}^{*}\right) k\left(x, x_{i}\right)+b
\end{array}
$$

where $k(\cdot)$ is kernel function, $a_{i}, a_{i}^{*}$, and $b$ are the parameters which could be obtained by the solution of the optimization problem (such problem is described in the form of (2)), and $c$ is the penalty factor.

SVM algorithm is sensitive to noise, and its calculation speed does not depend on the dimensions of input vectors but on the sample size. The large sample size will result in complex quadratic programming problem based on SVM, slow operational speed, and the long time that the calculation will spend. Therefore, LS-SVM is introduced on the basis of the traditional theory of SVM.

According to the theory of LS-SVM, (2) can be converted to the following linear equation:

$$
\left[\begin{array}{cc}
0 & \mathbf{A}_{h} \\
\mathbf{I} & \mathbf{\Omega}+\mathbf{C}^{-1} h
\end{array}\right]\left[\begin{array}{l}
\mathbf{b} \\
\mathbf{a}
\end{array}\right]=\left[\begin{array}{l}
0 \\
\mathbf{y}
\end{array}\right],
$$

where $\mathbf{y}=\left[y_{1}, y_{2}, \ldots, y_{h}\right]^{\mathrm{T}}, \mathbf{A}_{h}=[1,1, \ldots, 1], \mathbf{a}=$ $\left[a_{1}, a_{2}, \ldots, a_{h}\right]^{\mathrm{T}}, \mathbf{I}$ is the unit matrix, and $\boldsymbol{\Omega}_{i j}=\varphi\left(x_{i}\right) \varphi\left(x_{j}\right)=$ $k\left(x_{i}, x_{j}\right)$. Here, Gauss function is selected as Kernel function $k(\cdot)$ and it meets the following equation:

$$
\mathbf{K}\left(x_{k}, x_{h}\right)=\boldsymbol{\Phi}\left(x_{k}\right)^{\mathrm{T}} \boldsymbol{\Phi}\left(x_{h}\right) .
$$

Accordingly, (3) can be converted into the following equation:

$$
f(x)=\sum_{i=1}^{n} a_{i} k\left(x, x_{i}\right)+b .
$$

When LS-SVM is applied into the intelligent fitting of sieving efficiency $\eta$, the five-dimensional input vector is $\left\{\mathbf{a}, \mathbf{L}, \boldsymbol{\theta}, \mathbf{A}_{m}, \boldsymbol{\omega}\right\}$ and the one-dimensional output vector is $\{\boldsymbol{\eta}\}$. The 82 training samples come from the experimental results discussed above, and the prediction value of the LS-SVM model can be acquired finally by solving the value of $\alpha_{i}$ and $b$.

In the theory of LS-SVM, the parameters needed to be optimized are the penalty factor $c$ and the kernel parameter $r$, and the fitting performance of LS-SVM depends on the selection of $c$ and $r$.

Nowadays, there are many kinds of intelligent algorithm that can be used to optimize $c$ and $r$, such as particle swarm optimization algorithm, genetic algorithm, grid search, and chaos optimization algorithm [19, 20]. In these methods, genetic algorithm has been considered with increasing interest in a wide variety of applications, and it is widely used to solve linear and nonlinear problems by exploring all regions of state space and exploiting potential areas through mutation, crossover, and selection operations applied to individuals in the population. In this paper, adaptive genetic algorithm is applied to determine the value of $c$ and $r$.

\section{Intelligent Prediction of Sieving Efficiency Based on LS-SVM}

5.1. Optimization of Parameters by Adaptive Genetic Algorithm. The key of adaptive genetic algorithm [21] is to determine the fitness function. Here, it is described as follows:

$$
F(c, r)=\frac{1}{\sum_{i=1}^{n}\left[y_{i}-f\left(x_{i}\right)\right]^{2}+e},
$$

where $y_{i}$ is the expected output, $f\left(x_{i}\right)$ is the actual output, and $e$ is a small real number, which is to prevent the situation of zero denominator; here $e=10^{-3}$.

The initial crossover probability and the initial mutation probability are determined by

$$
\begin{aligned}
& P_{c}^{1}= \begin{cases}0.9-0.3 \frac{f^{\prime}-f_{\mathrm{avg}}}{f_{\max }-f_{\mathrm{avg}}}, & f \geq f_{\mathrm{avg}} \\
0.9 & f<f_{\mathrm{avg}},\end{cases} \\
& P_{m}^{1}= \begin{cases}0.1-0.099 \frac{f_{\max }-f}{f_{\max }-f_{\mathrm{avg}}}, & f \geq f_{\mathrm{avg}} \\
0.1 & f<f_{\mathrm{avg}},\end{cases}
\end{aligned}
$$

where $f^{\prime}$ is the larger fitness function value of the cross two bodies, $f$ is the fitness function value of individual, $f_{\text {avg }}$ is the average fitness function value in the samples, and $f_{\max }$ is the maximum fitness function value of individual in the samples.

Crossover probability and mutation probability change with the evolutional generation, and their changing rules are as follows:

$$
\begin{aligned}
& P_{c}^{t}= \begin{cases}0.9 \sqrt{1-\left(\frac{t}{t_{\max }}\right)^{2},} & P_{c}^{t}<0.6 \\
0.6 & P_{c}^{t} \geq 0.6\end{cases} \\
& P_{m}^{t}= \begin{cases}0.1 e^{\left(-\lambda t / t_{\max }\right)}, & P_{m}^{t}<0.001 \\
0.001 & P_{m}^{t} \geq 0.001,\end{cases}
\end{aligned}
$$

where $t$ is the genetic algebra, $t_{\max }$ is the terminated genetic algebra, and $\lambda$ is a constant; here $\lambda=10$. 
The basic steps of "adaptive genetic algorithm" are listed as follows.

Step 1. Generate initial population $P(0)$; algebra is set to 0 , and the number of individuals is $M$.

Step 2. Run selection operator, crossover operator, and mutation operator in proper order and calculate the fitness of individuals.

Step 3. Sort individuals by fitness value and run the intelligent fitting model of LS-SVM once for the individual which has highest fitness value.

Step 4. Get a new generation of population $P(t+1)$, and algebra increases by 1 .

Step 5. Judge whether it meets the optimization criterion. If it meets the criteria, the optimization process is over; else, return to Step 2.

There are two factors that may lead to the failure of LSSVM: (1) "overfitting" issue (the objective function can reduce to a very low value during the training phase, but the testing error is relatively large); (2) "underfitting" issue (the objective function cannot reduce to a low value during the training phase). For solving these issues, "cross-validation" method is introduced $[22,23]$.

The basic steps of "cross-validation" are listed as follows.

Step 1. Divide the training samples into $n$ subsamples and set $i=1$.

Step 2. Regard the $i$ th subsamples as the testing samples and the others as the training samples.

Step 3. Optimize the parameters $\left(c_{i}, r_{i}\right)$ in LS-SVM based on the new training samples by adaptive genetic algorithm.

Step 4. $i=i+1$, and repeat Steps $1-3$ till $i=n$ and $c=\sum c_{i} / n$, $r=\sum r_{i} / n$.

In this paper, the population size is 40 , the dimension of parameters to be optimized is 2 , the maximum genetic algebra is 400 , and the binary digit of each variable is 20 . And after the optimization process, $c \approx 55$ and $r \approx 1.2$. Error function is defined as follows:

$$
f_{E}=\frac{1}{n} \sum_{i=1}^{n} \frac{\left|f\left(x_{i}\right)-y_{i}\right|}{y_{i}},
$$

where $f\left(x_{i}\right)$ is the actual output and $y_{i}$ is the expected output.

5.2. Comparison of the LS-SVM Model, the Existing Formula, and Neural Network. An existing fitting formula of sieving efficiency from literature [10] is shown as follows:

$$
\eta=80.76+2.51 a-3.32 \beta,
$$

where $a$ is screen mesh size, $\beta$ is screen deck angle, and $\eta$ is sieving efficiency.

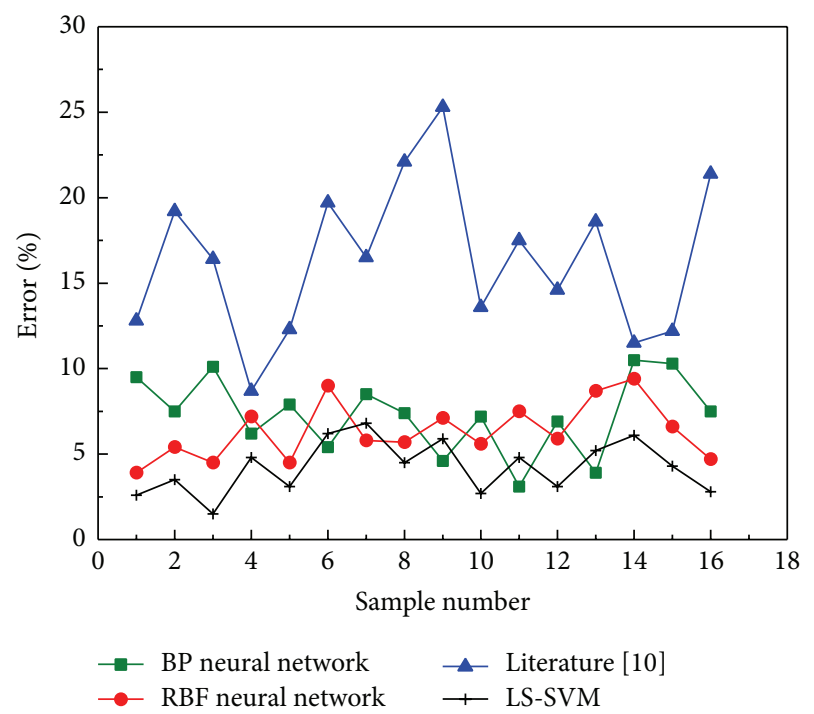

FIGURE 8: Fitting error comparison of the LS-SVM model, literature [10], and neural network.

Neural network is also a usual intelligent model to fit the numerical relation besides LS-SVM; the training points in LS-SVM are still selected as training samples to optimize the parameters in neural network. The training methods of neural network have detailed descriptions in literature [24]. The prediction error between LS-SVM, BP neural network, RBF neural network, and the existing fitting formula in literature [10] is shown in Figure 8.

Figure 8 shows that the prediction error of selected 16 testing samples is analyzed; the average relative error of $\mathrm{BP}$ neural network is $7.3 \%$, the average relative error of RBF neural network is $6.4 \%$, the average relative error of literature [10] is $16.2 \%$, and the average relative error of LS-SVM model is $4.2 \%$. So the prediction performance of LS-SVM model is better than that of neural network and the existing fitting formula in literature [10].

\section{Conclusions}

The effects of different factors on sieving efficiency were investigated based on the vibration sieving test bench. According to experimental results, the sieving efficiency $\eta$ increases with the increase of vibration amplitude $A_{m}$ and screen length $L$, and it first increases and then decreases with the increase of vibration frequency $\omega$, mesh aperture size $a$, and inclination angle $\theta$.

The intelligent model to predict the sieving efficiency was established based on the experimental results, LS-SVM theory, and the adaptive genetic algorithm. The average prediction error of the LS-SVM model examined by testing points can be reduced to $4.2 \%$, which is much less than that of neural network and the existing fitting formula. So, the LSSVM model has a better prediction performance on sieving efficiency, which provides a theoretical basis for the optimal design and parameters selection of sieving screens. 


\section{Competing Interests}

The authors declare that there is no conflict of interests regarding the publication of this paper.

\section{Acknowledgments}

The authors thank the National Science Foundation of China (nos. 51276056 and 51305045) for financial support.

\section{References}

[1] J. Li, C. Webb, S. S. Pandiella, and G. M. Campbell, "Discrete particle motion on sieves-a numerical study using the DEM simulation," Powder Technology, vol. 133, no. 1-3, pp. 190-202, 2003.

[2] K. J. Dong, A. B. Yu, and I. Brake, "DEM simulation of particle flow on a multi-deck banana screen," Minerals Engineering, vol. 22, no. 11, pp. 910-920, 2009.

[3] K. S. Liu, "Some factors affecting sieving performance and efficiency," Powder Technology, vol. 193, no. 2, pp. 208-213, 2009.

[4] G. W. Delaney, P. W. Cleary, M. Hilden, and R. D. Morrison, "Testing the validity of the spherical DEM model in simulating real granular screening processes," Chemical Engineering Science, vol. 68, no. 1, pp. 215-226, 2012.

[5] H. G. Jiao and Y. M. Zhao, "Screen simuliation using a particle discrete element method," Journal of China University of Mining and Technology, vol. 36, no. 2, pp. 232-236, 2007.

[6] H. C. Li, Y. M. Li, and Z. Tang, "Numerical simulation and analysis of vibration screening based on EDEM," Transactions of the Chinese Society of Agriculture Engineering, vol. 27, no. 5, pp. 117-121, 2011.

[7] Z. Z. Shi and Y. J. Huang, "Research on screening efficiency based on AR model of high-order cumulant LS-SVM," China Mechanical Engineering, vol. 22, no. 16, pp. 1965-1969, 2011.

[8] V. Grozubinsky, E. Sultanovitch, and I. J. Lin, "Efficiency of solid particle screening as a function of screen slot size, particle size, and duration of screening," International Journal of Mineral Processing, vol. 52, no. 4, pp. 261-272, 1998.

[9] Y. H. Chen and X. Tong, "Modeling screening efficiency with vibrational parameters based on DEM 3D simulation," Mining Science and Technology, vol. 20, no. 4, pp. 615-620, 2010.

[10] H. G. Jiao, J. R. Li, and Y. M. Zhao, "Test and research on optimum configuration of diameter of screen aperture and incline of screen deck," Coal Preparation Technology, vol. 35, no. 2, pp. 1-4, 2007.

[11] E. Jia-Qiang, Intelligent Fault Diagnosis and Its Application, Hunan University Press, Changsha, China, 2006.

[12] S. Umehara, T. Yamazaki, and Y. Sugai, "A precipitation estimation system based on support vector machine and neural network," Electronics and Communications in Japan, vol. 89, no. 3, pp. 38-47, 2006.

[13] A. Kuh and P. De Wilde, "Comments on pruning error minimization in least squares support vector machines," IEEE Transactions on Neural Networks, vol. 18, no. 2, pp. 606-609, 2007.

[14] K. Lamorski, Y. Pachepsky, C. Sławiński, and R. T. Walczak, "Using support vector machines to develop pedotransfer functions for water retention of soils in Poland," Soil Science Society of America Journal, vol. 72, no. 5, pp. 1243-1247, 2008.
[15] C. H. Wang, Z. P. Zhong, R. Li, and E. Jia-Qiang, "Prediction of jet penetration depth based on least square support vector machine," Powder Technology, vol. 203, no. 2, pp. 404-411, 2010.

[16] C. H. Wang, Z. P. Zhong, R. Li, and J. Q. E, "Intelligent fitting of minimum spout-fluidised velocity in spout-fluidised bed," Canadian Journal of Chemical Engineering, vol. 89, no. 1, pp. 101107, 2011.

[17] J. E, C. Qian, T. Liu, and G. Liu, "Research on the vibration characteristics of the new type of passive super static vibratory platform based on the multiobjective parameter optimization," Advances in Mechanical Engineering, vol. 2014, Article ID 569289, pp. 1-8, 2014.

[18] L. J. Dong, X. B. Li, M. Xu, and Q. Li, “Comparisons of random forest and support vector machine for predicting blasting vibration characteristic parameters," Procedia Engineering, vol. 26, pp. 1772-1781, 2011.

[19] E. Jiaqiang, C. Qian, H. Liu, and G.-L. Liu, "Design of the $H_{\infty}$ robust control for the piezoelectric actuator based on chaos optimization algorithm," Aerospace Science and Technology, vol. 47, pp. 238-246, 2015.

[20] E. Jiaqiang, C. Qian, T. Liu, and G. Liu, "Chaos analysis on the acceleration control signals of the piezoelectric actuators in the stewart platform," Shock and Vibration, vol. 2016, Article ID 8087176, 9 pages, 2016.

[21] K. P. Ferentinos and T. A. Tsiligiridis, "Adaptive design optimization of wireless sensor networks using genetic algorithms," Computer Networks, vol. 51, no. 4, pp. 1031-1051, 2007.

[22] L. J. Dong, X. B. Li, and G. N. Xie, "Nonlinear methodologies for identifying seismic event and nuclear explosion using random forest, support vector machine, and naive bayes classification," Abstract and Applied Analysis, vol. 2014, Article ID 459137, 8 pages, 2014.

[23] L. J. Dong and X. B. Li, "Comprehensive models for evaluating rockmass stability based on statistical comparisons of multiple classifiers," Mathematical Problems in Engineering, vol. 2013, Article ID 395096, 9 pages, 2013.

[24] J. Q. E, H. Y. Zuo, and Z. Q. Luo, Fuzzy Inference Neural Network Intelligent Information Fusion and Its Engineering Application, China Water Power Press, Beijing, China, 2012. 


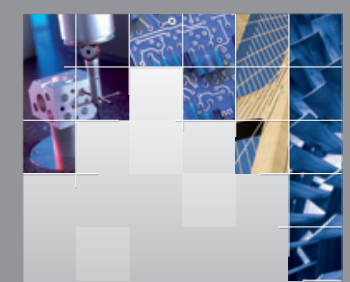

\section{Enfincering}
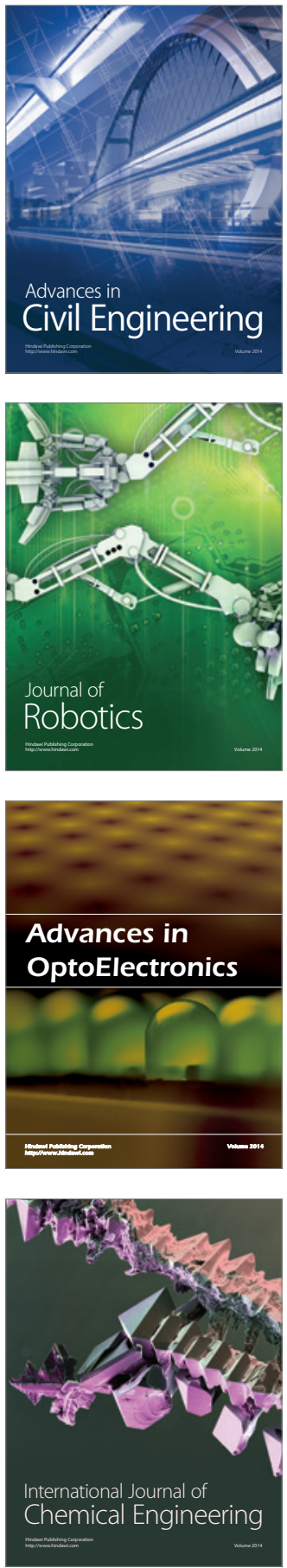

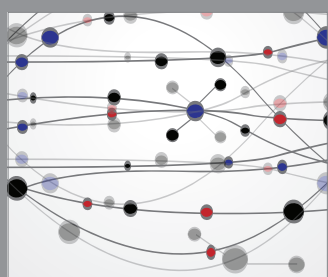

The Scientific World Journal

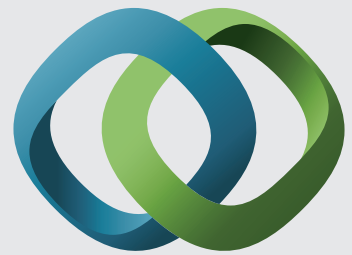

\section{Hindawi}

Submit your manuscripts at

http://www.hindawi.com
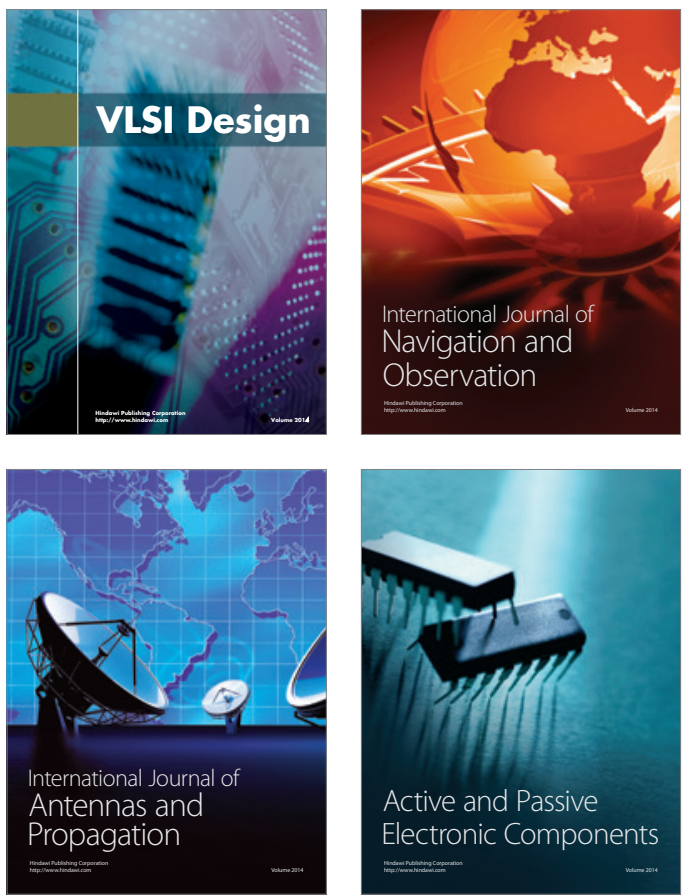
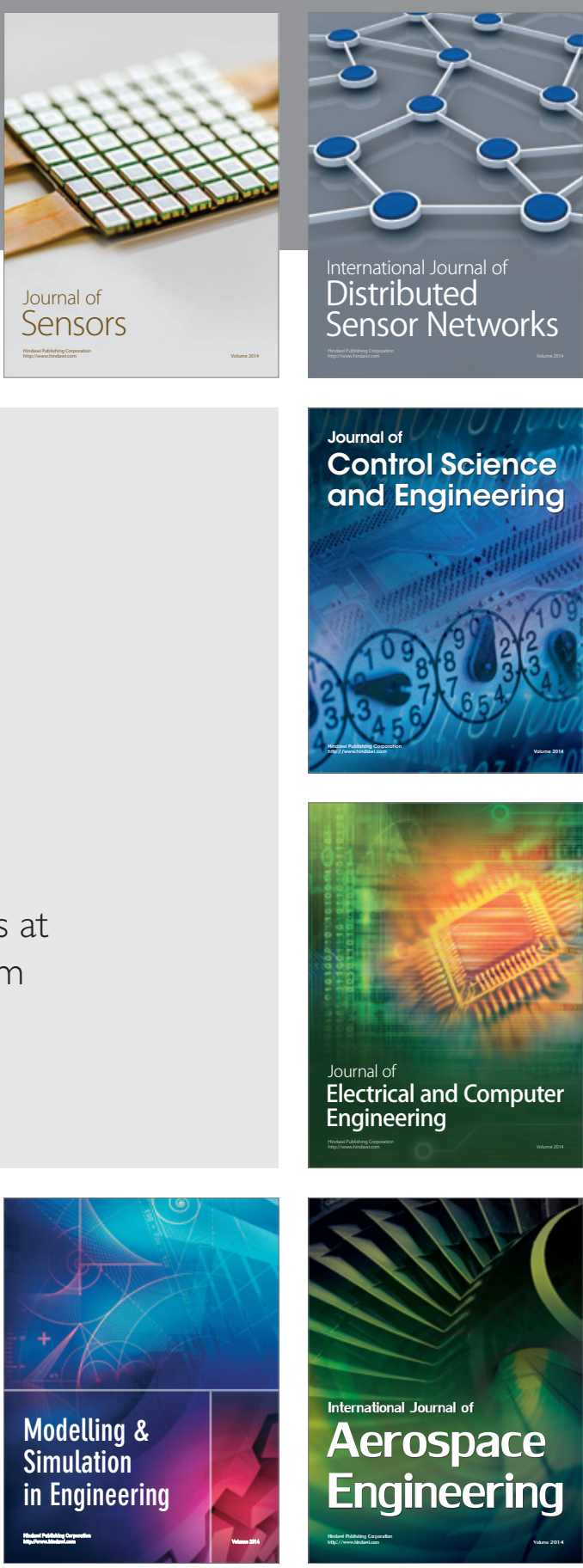

International Journal of

Distributed

Sensor Networks

Journal of

Control Science

and Engineering
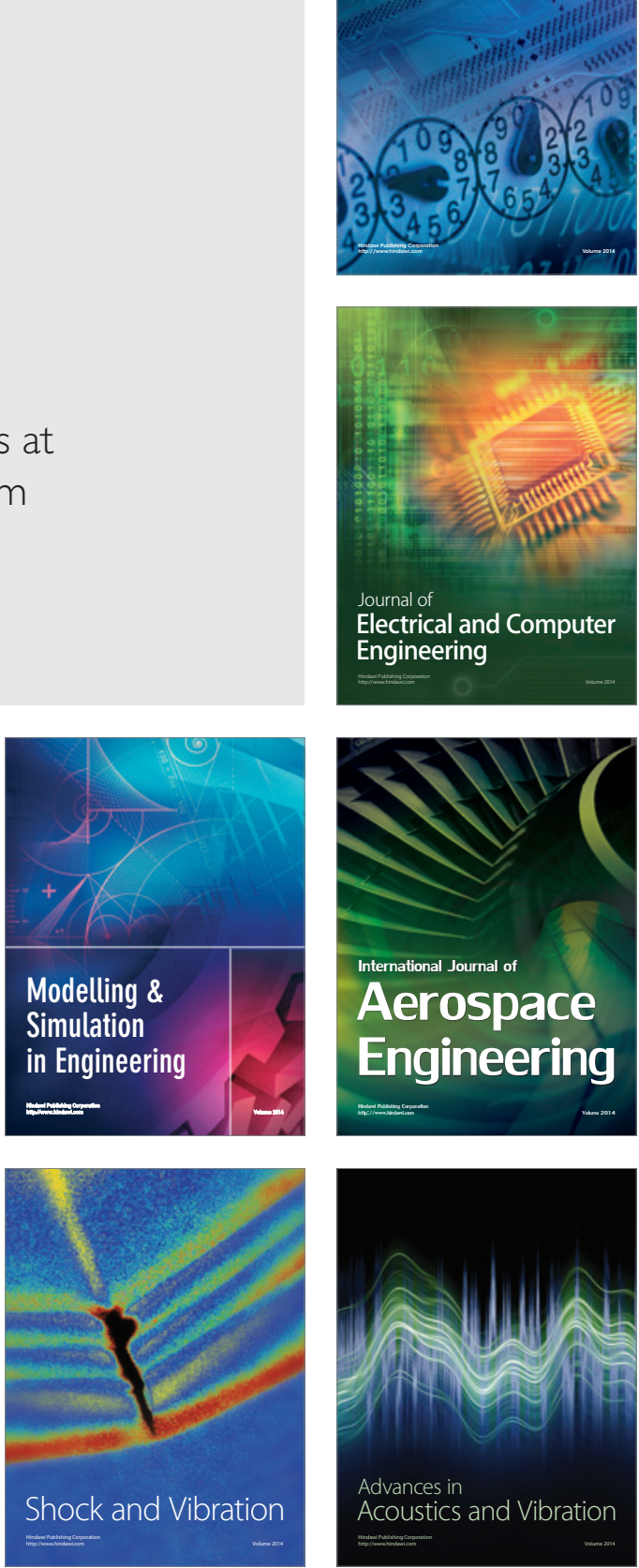